\title{
Influence of Vehicle Sound Source Height on Traffic Noise Forecast and Sound Barrier Design
}

\author{
Xintan $\mathrm{Ma}^{\mathrm{a}}$, Guopeng Chang ${ }^{\mathrm{b}}$ \\ School of Vehicle and Transportation Engineering, Henan University of Science and Technology, \\ Henan Luoyang 471003, China \\ a amaxintan@163.com \\ b2468518077@qq.com
}

Keywords: Noise model, Sound source height, Sound barrier, Sound pressure level.

\begin{abstract}
At present ,the prediction result of highway traffic noise is quite different from the actual situation ,the main reason of which is that the prediction model of the sound source is not perfect .This paper considers the actual height of highway sound source, uses acoustic simulation software to build a sound source model whose vertical height is consistent with the actual situation, analysis the prediction results of sound receiving points and discovers that: compared with the equivalent simple sound source model, that having considered the sound source height much more accord with the actual highway situation so that the prediction accuracy will be improved; the difference value of the prediction results is the most prominent under three meters of barrier when the road traffic condition are all heavy vehicles and the comprehensive noise difference of the two sound source models is $1.71 \mathrm{~dB}$, it will reach difference value of spectrum peak for $3.03 \mathrm{~dB}$ when the frequency is $315 \mathrm{HZ}$, which will provide reference to the road traffic noise control.
\end{abstract}

\section{Introduction}

With the increasing of car ownership, the traffic noise pollution has caused a deep disturbance to our life, so the prevention and control of road noise pollution is urgent. Road traffic noise model is the basis of road noise research. Documentation [1-2] The prediction results and measurement of FHWA, RLS 90 and RLS model were analyzed, and the error of noise prediction was larger when heavy vehicles were more frequent. The noise prediction model is improved based on FHWA. Documentation [3] the absolute error of diurnal pressure level prediction in the guideline model was found to be 3.3-3.6 dB and-3.4-0.29 dB in the urban level road. In the current traffic noise model, a linear sound source model near the ground along the equivalent line is used to simulate the road traffic sound source (also known as the equivalent single sound source model [4]), The equivalent sound source model neglects the influence of the actual height of sound source on the definition of sound source and the prediction of sound pressure level of road noise model.

Based on the equivalent sound source model, this paper considers the actual height of the sound source in three kinds of road conditions: heavy vehicle, light vehicle and light, medium and heavy vehicle, establishes the traffic noise model in the same direction as the sound source, calculates the comprehensive noise and sound pressure level of the sound point in three kinds of road conditions, and compares the prediction results of the equivalent sound source under the same conditions.

\section{Acoustic Source and Sound Barrier Insertion Loss}

Only when the sound source model is close to the actual condition of the road can the sound pressure level prediction at the sound point be more accurate. At home and abroad, the processing method of noise source model is divided into light vehicle, medium vehicle and heavy vehicle. The height of sound source is $0.35 \mathrm{~m}, 0.85 \mathrm{~m}$ and $1.5 \mathrm{~m}$, respectively. In order to calculate the average height of noise source for convenient traffic, the whole body is $1 \mathrm{~m} \mathrm{[5].} \mathrm{The} \mathrm{location} \mathrm{of} \mathrm{the} \mathrm{sound}$ source is determined by means of the equivalent driving line, located near the road center line. If the vehicle traffic volume is greater than 1000 hours, the road traffic sound source can be regarded as an 
online sound source [6]. According to the road noise measurement method, the sound pressure level spectrum from the equivalent driving line $r_{0}$ is obtained, and then according to the relationship between the sound pressure level and the power level of the ambient noise standard, the sound pressure level and the power of the sound level of the noise is obtained [7].

In practice, road noise can be regarded as the noise model of finite long-term sound source and finite-long sound barrier. When calculating, the noise attenuation is calculated according to the infinite long sound source and the infinite long sound barrier. Then the noise decay reduction is corrected according to the shielding angle of the sound barrier $(\beta / \theta)$.

$$
\Delta L_{d}=\left\{\begin{array}{cc}
10 \lg \left[\frac{3 \pi \sqrt{\left(1-t^{2}\right)}}{4 \arctan \sqrt{\frac{1-t}{1+t}}}\right] & t=\frac{40 f_{i} \delta}{3 c} \leq 1 \\
10 \lg \left[\frac{3 \pi \sqrt{t^{2}-1}}{2 \ln \left(t+\sqrt{t^{2}-1}\right)}\right] & t=\frac{40 f_{i} \delta}{3 c}>1
\end{array}\right.
$$

$f_{i}$-The central frequency of sound waves in a third of the frequency range of $\mathrm{i}, \mathrm{Hz} ; \delta$-Sound path differences, $\mathrm{m}$; C-Sound speed, $\mathrm{m} / \mathrm{s}$;

The sound attenuation of the finite long sound barrier is amended to $\Delta L_{i}$ depending on the size of the shielding angle $(\beta / \theta)$.Acoustic pressure level of the sound point behind the sound barrier $L_{p}$ :

$$
L_{p}=10 \lg \sum 10^{0.1}\left(L_{p_{i}}-\Delta L_{i}\right)
$$

$L_{p_{i}}$-Sound pressure level of reference point $f_{i}$ in silent barrier; $\Delta L_{i}$-Emission reduction after correction of the finite sound barrier

\section{Sound Source Model}

It is very important to determine and define the location of the traffic sound source. In the past research, traffic noise is regarded as all along the road direction, and the actual height of the sound source is ignored.

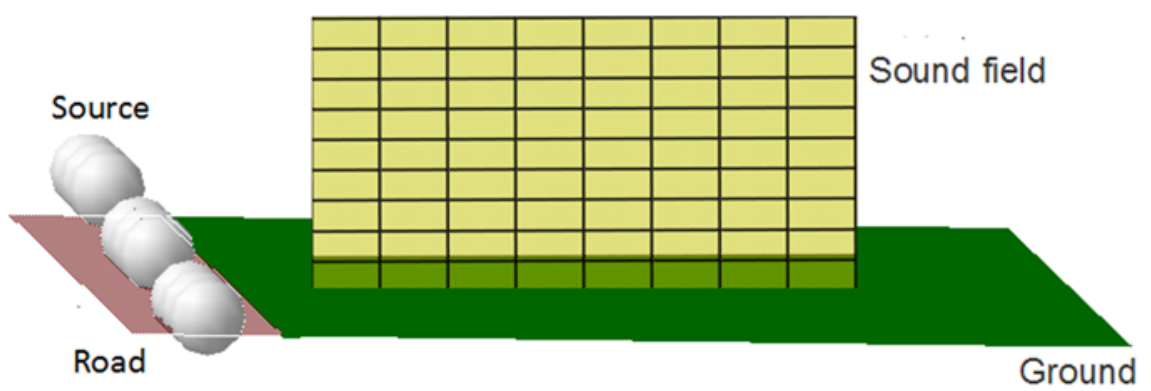

Figure 1. Traffic noise model without a sound barrier

Establishment of a road model $14.5 \mathrm{~m}$ in length with a distance of $30 \mathrm{~m}$ between vehicles and 300 $\mathrm{m}$ [8].According to three kinds of road calculation schemes: scheme 1, all heavy vehicle road, choose 9 points to define the source, source height $1.5 \mathrm{~m}$; Option 2, all light vehicle road, choose 9 points to define the source, source height $0.35 \mathrm{~m}$; Option 3, light, medium and heavy vehicles mixed in the road, selected the source height of $0.35 \mathrm{~m}, 0.85 \mathrm{~m}, 1.5 \mathrm{~m}$ point respectively; When all three models exist, the three-source model is at $20 \mathrm{~m}$ from the shoulder and $2 \mathrm{~m}$ high, as shown in Figure 1. According to relevant criteria [8] the frequency response curve from the equivalent driving line $r_{0}=$ $7.5 \mathrm{~m}$ is shown in Figure 2. 


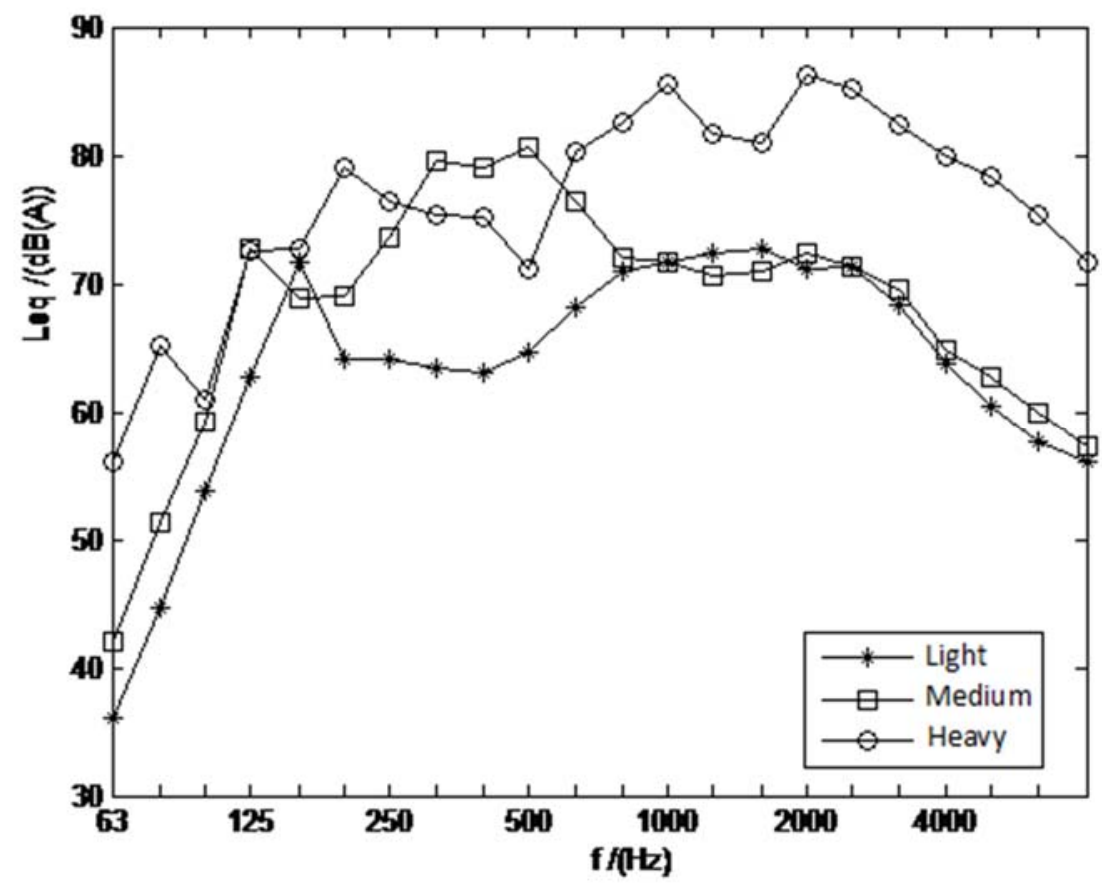

Figure 2. The sound pressure level spectrum of different vehicle models

Having obtained the sound pressure level value of the reference point, using the formula(5) to obtain the sound pressure level spectrum of the three vehicle acoustic source locations through the sound pressure level spectrum of the reference point, and using the formula(2) to obtain the sound power level spectrum of the light, medium and heavy vehicle acoustic model, as shown in table 1, and defining parameters such as air temperature, spectrum absorption characteristics of the ground In Virtual. lab. the frequency spectrum of sound sources of light, medium and heavy vehicles at the site of sound is calculated.

Table 1. The Sound Power Level of Different Vehicle Sound Sources

\begin{tabular}{crcc}
\hline $\begin{array}{c}\text { 1/3 } \\
\text { Octave(Hz) }\end{array}$ & $\begin{array}{c}\text { Light Vehicle } \\
\text { Sound } \\
\text { Power Level } \\
\text { dB(A) }\end{array}$ & $\begin{array}{c}\text { Medium Vehicle sound power level } \\
\text { dB(A) }\end{array}$ & $\begin{array}{c}\text { Heavy Vehicle sound power level } \\
\text { dB(A) }\end{array}$ \\
\hline 63 & 47.75 & 53.75 & 67.55 \\
125 & 74.25 & 84.25 & 84.25 \\
160 & 83.33 & 80.50 & 84.25 \\
200 & 75.92 & 81.83 & 90.50 \\
315 & 75.08 & 91.17 & 86.92 \\
400 & 74.67 & 90.75 & 86.75 \\
800 & 82.58 & 83.58 & 94.25 \\
1250 & 84.17 & 82.17 & 93.43 \\
1600 & 84.25 & 82.58 & 92.53 \\
2000 & 82.67 & 83.38 & 97.83 \\
3150 & 80.08 & 80.92 & 94.08 \\
4000 & 75.50 & 76.58 & 91.67 \\
\hline
\end{tabular}

The acoustic pressure level spectrum at the same sound point $(\mathrm{r}=20 \mathrm{~m})$ is calculated by using the sound pressure level superposition formula of three kinds of vehicles.

\section{Simulation Results and Analysis}

\subsection{No sound Barrier}

In the absence of a sound barrier, the combined noise of the model considering the source height and the equivalent sound source model is $93.27 \mathrm{~dB}$ and $92.04 \mathrm{~dB}$ respectively, the combined noise is $1.23 \mathrm{~dB}$, the difference between the acoustic pressure at the point and the acoustic pressure level is $15701 \mathrm{~dB}$ in the frequency range (63-4000), and the difference between the sound pressure at the 
point and the sound pressure is $0.97 \mathrm{~dB}-1.77 \mathrm{~dB}$. A difference greater than $1000 \mathrm{~Hz}$ tends to be stable to about $1 \mathrm{~dB}$, the spectral difference is shown in Figure 4.

Table 2. The equivalent acoustic power level of a single source model

\begin{tabular}{cccccc}
\hline $\begin{array}{c}\text { 1/3Octave } \\
(\mathbf{H z})\end{array}$ & $\begin{array}{c}\text { Sound Power Level } \\
\mathbf{d B}(\mathbf{A})\end{array}$ & $\begin{array}{c}\mathbf{1 / 3 O c t a v e} \\
\mathbf{( H z )}\end{array}$ & $\begin{array}{c}\text { Sound Power Level } \\
\mathbf{d B}(\mathbf{A})\end{array}$ & $\begin{array}{c}\mathbf{1 / 3 O c t a v e} \\
(\mathbf{H z})\end{array}$ & $\begin{array}{c}\text { Sound Power Level } \\
\mathbf{d B}(\mathbf{A})\end{array}$ \\
\hline 63 & 67.78 & 315 & 92.46 & 1250 & 94.20 \\
80 & 76.99 & 400 & 92.29 & 1600 & 93.55 \\
100 & 75.33 & 500 & 92.74 & 2000 & 98.14 \\
125 & 87.48 & 630 & 93.49 & 2500 & 97.11 \\
160 & 87.74 & 800 & 94.88 & 3150 & 94.46 \\
200 & 91.09 & 1000 & 97.83 & 4000 & 91.91 \\
250 & 90.03 & & & & \\
\hline
\end{tabular}

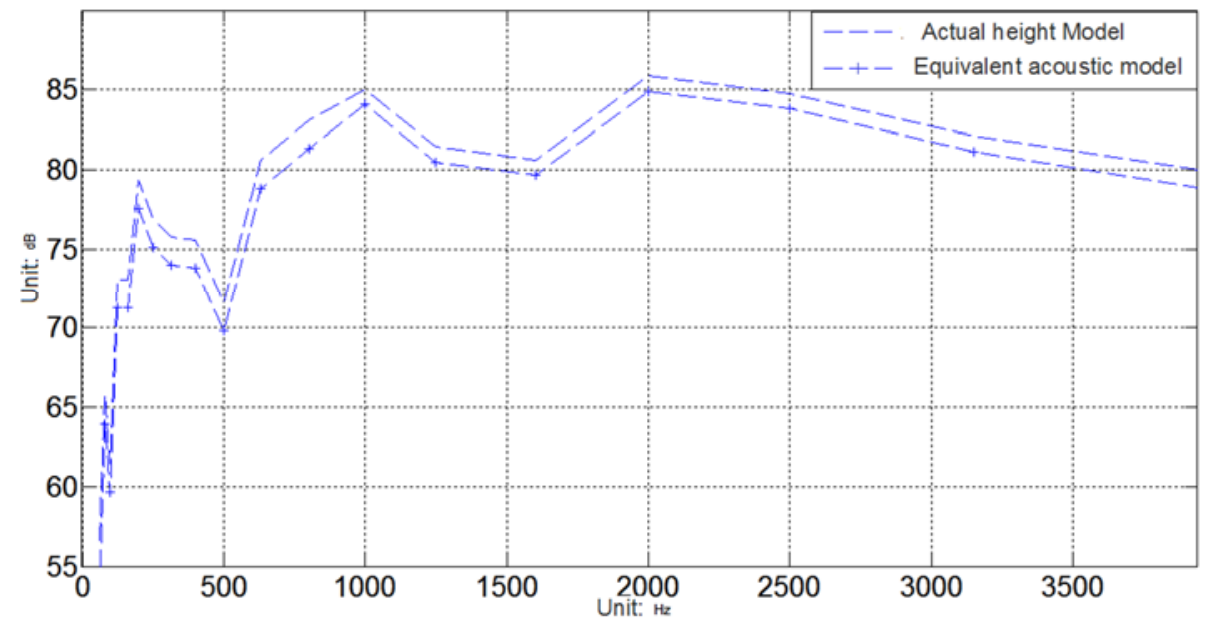

Figure 3. The sound pressure level frequency response curve of heavy traffic condition without sound barrier

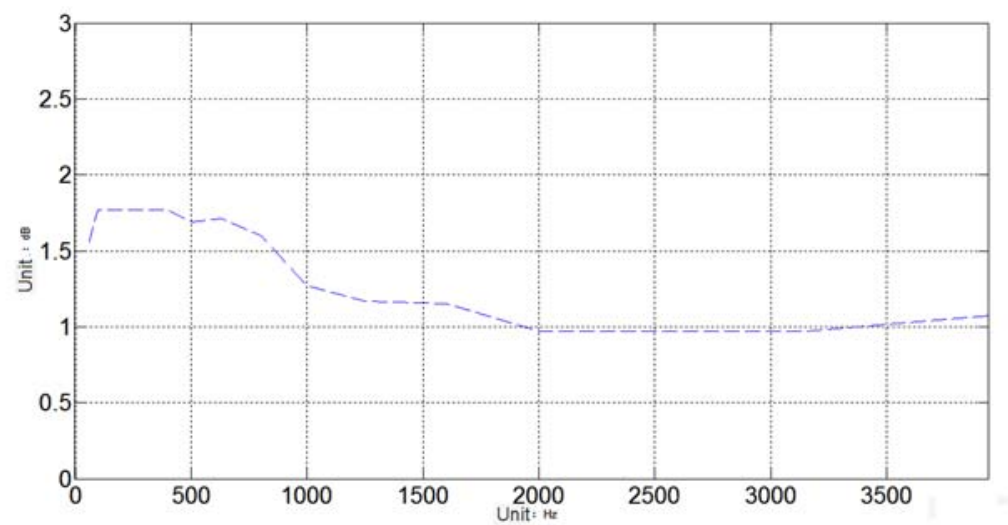

Figure 4. The spectral difference of the acoustic pressure level of two acoustic source models without sound barrier

When all light vehicle road conditions are used, the combined noise of the model considering the sound source height and the equivalent sound source model is $77.62 \mathrm{~dB}$ and $78.48 \mathrm{~dB}$ respectively, the difference of the combined noise is $0.86 \mathrm{~dB}$ and the difference of sound pressure level at different frequencies is $0.6 \mathrm{~dB}-1.5 \mathrm{~dB}$; At $80 \mathrm{~Hz}$ is the maximum margin of $1.89 \mathrm{db}$. When the road conditions exist for light, medium and heavy vehicles, the combined noise of the three-source model and the equivalent source model are $89.12 \mathrm{~dB}$ and $88.67 \mathrm{~dB}$ respectively, the difference of the combined noise is $0.35 \mathrm{~dB}$ and the difference of the sound pressure level of the receiving point is $0.05 \mathrm{~dB}-0.42$ $\mathrm{db}$. 


\subsection{Insert Sound Barrier}

The length of the sound barrier is $120 \mathrm{~m}$ [ 9] The other parameters remain unchanged. When the road traffic condition is all heavy vehicles, the combined noise of the model considering the source height is $84.74 \mathrm{~dB}$ and the equivalent source model is $83.03 \mathrm{~dB}$ respectively, the combined noise difference is $1.71 \mathrm{~dB}$, the noise difference is $2.4 \mathrm{~dB}-3.03 \mathrm{~dB}$ within the frequency range (63-4000) of the sound pressure of the acoustic point and reaches a peak of $3.03 \mathrm{~dB}$ at $400 \mathrm{~Hz}$; The margin decreases over $1000 \mathrm{~Hz}$.

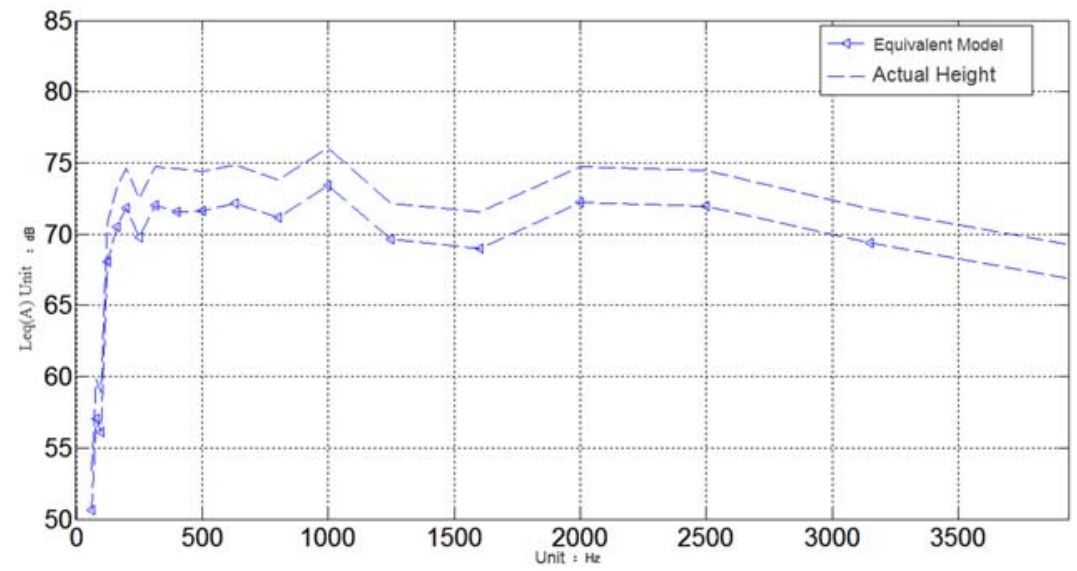

Figure 5. The sound pressure level frequency response curve of heavy traffic condition with sound barrier

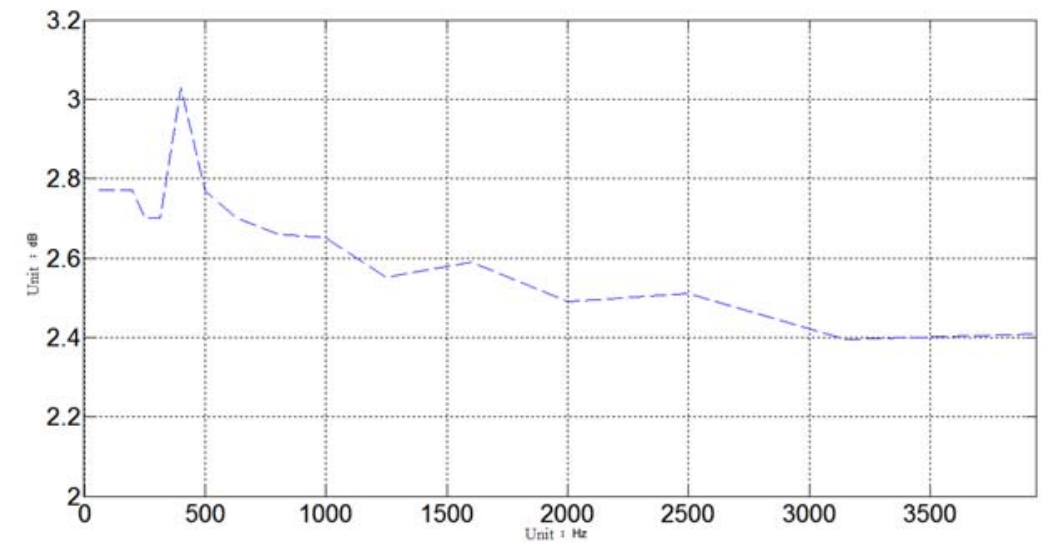

Figure 6. The spectral difference of the acoustic pressure level of two acoustic source models with sound barrier

When all light vehicle road conditions are concerned, the synthetical noise of the sound source height model and the equivalent sound source model is $70.94 \mathrm{~dB}$ and $72.15 \mathrm{~dB}$ respectively, the difference of the synthetical noise is $1.21 \mathrm{db}$. When light, medium and heavy vehicles are present in the road, the integrated noise of the three-source model is $81.91 \mathrm{~dB}$, that of the single source is 81.64 $\mathrm{dB}$, and the integrated noise difference is $0.27 \mathrm{db}$. The spectral difference between the three-source model and the equivalent source model is $0 \mathrm{~dB}-1.53 \mathrm{~dB}$, with a peak difference of $1.53 \mathrm{~dB}$ at $160 \mathrm{~Hz}$, and between $0-0.4 \mathrm{~dB}$ at the end of $2000 \mathrm{~Hz}$.

\section{Conclusion}

The height of sound source is an important factor affecting the accuracy of the sound source model, which directly influences the frequency spectrum of the sound pressure level and the prediction of the integrated noise. It is more accurate to establish a sound source model which is consistent with the actual situation of the sound source.

When the road is all light or heavy vehicles, considering the source height model and the equivalent source prediction results are more different, especially when there are more heavy vehicles or all heavy vehicles. This is because the equivalent sound source model is close to the actual condition of 
the road when the light, medium and heavy vehicles are mixed. When the road is full of light vehicles or heavy vehicles, the equivalent acoustic source model is different from that of the road, which indicates that the equivalent single source model is reasonable under certain conditions.

In the case of the silent barrier, the difference between the prediction results of the model considering the actual height of the acoustic source and the equivalent single source model is 0.35 $\mathrm{dB}-1.23 \mathrm{~dB}$. In the case of sound barrier, the difference between the model considering the actual height of the sound source and the equivalent single source model is $0.27 \mathrm{~dB}-1.7 \mathrm{~dB}$; the combined noise difference between the two models was $1.71 \mathrm{~dB}$ for all heavy vehicle road conditions and 3.03 $\mathrm{dB}$ for the acoustic pressure spectrum at $315 \mathrm{~Hz}$ which was significant.

\section{References}

[1]. Cao Li-na, Shang Yong, Lu Lin-guo. Comparison of FHWA, RLS90 and MOT Model for Highway Traffic Noise Prediction[J]. Journal of Zhengzhou University (Engineering Science), 2014,35(3): 106-110.

[2]. Li Na Cao, Wei Sheng Guan, Application of An Improved Noise Prediction Model Based on FHWA in Environmental Impact Assessment of Shang Zhou to Dan Feng Expressway[J]. Applied Mechanics and Materials,2013,409-410,688-694.

[3]. Fan Dong-ping. Road traffic noise prediction model analysis and empirical research [D]. Guangzhou: Guangdong University of Technology,2012:14-15.

[4]. Ma Xin-tan, Feng Shi-chang. Simulation and Analysis on the Traffic Noise Models of Four-Lane Highway[J]. Machinery Design \& Manufacture, 2017, (z1):106-109.

[5]. Zhang Yu-fen, Qian Bing-hua, Dai Ming-xin. Transportation and Environmental Protection[M]. Beijing: China Communications Press,2003:106-108.

[6]. Zhao Chun-lai, Ma Xin-tan, Guo Zhi-jun. Optimization Design of Highway Sound Barrier Based on the Effective Length of Sound Source[J]. Noise and Vibration Control,2011(1):153-156.

[7]. Zhao Chun-lai, Ma Xin-tan, Guo Zhi-jun. Parametric Analysis and Optimization Design of Road Noise Barrier[J]. Journal of Henan University of Science \& Technology (Natural Science), 2010, 31(4):23-27.

[8]. GB3096-2008, Environmental quality standard for noise[S].4-6.

[9]. Li Lei. Study on Doppler Effect of Vehicle Noise and the Optimization Method of Noise Barrier [D]. Henan University of Science and Technology, 2015:30-31. 